\title{
Ácido tânico como agente biomodificador de dentina
}

\section{Tanic acid as biomodificator of collagen dentin}

\author{
Talita Arrais Daniel Mendes ${ }^{1,2}$ (D), Samuel Chillavert Dias Pascoal ${ }^{3}$ (D), Marcelo Victor Sidou Lemos ${ }^{1}$ (D), Sérgio Lima Santiago ${ }^{4}$ (D), \\ Juliano Sartori Mendonça ${ }^{4}$ (D)
}

1. Doutoranda pelo Programa de Pós-Graduação em Odontologia da Universidade Federal do Ceará (UFC), Fortaleza, CE, Brasil. 2. Docente do Curso de Odontologia do Centro Universitário Unicatólica (Unicatólica), Quixadá, CE, Brasil. 3. Discente do Curso de Odontologia pela Universidade Federal do Ceará (UFC), Fortaleza, CE, Brasil. 4. Docente do Programa de Pós-Graduação em Odontologia da Universidade Federal do Ceará (UFC), Fortaleza, CE, Brasil

\begin{abstract}
Resumo
Introdução: a utilização de diferentes polifenóis no aumento das propriedades mecânicas do colágeno dentinário tem sido bastante empregada, sendo o ácido tânico um polifenol com propriedade biológica bastante pronunciada. Objetivo: avaliar o potencial biomodificador do ácido tânico (AT) em diferentes concentrações em colágeno dentinário. Métodos: os fatores sob investigação foram módulo de elasticidade, mensurado por meio de um ensaio de flexão de três pontos $(n=10)$ e variação de massa $(n=10)$, aferidos com uma balança de precisão, sendo, para tanto, avaliados os seguintes elementos: ácido tânico $(0,1 ; 1$ e $10 \%)$, proantocianidina (6,5\%) e água destilada (controle). Resultados: quanto ao módulo de elasticidade, os grupos tratados com ácido tânico apresentaram valores, estatisticamente, superiores $(p<0,05)$ aos demais grupos, não sendo observadas diferenças entre suas diferentes concentrações. Para os valores de variação de massa, os grupos que foram imersos em agentes biomodificadores apresentaram valores estatisticamente $(p<0,05)$ superiores ao grupo controle. Conclusão: o ácido tânico se apresenta como um potencial agente biomodificador do colágeno dentinário, independente da concentração utilizada, aumentando o seu módulo de elasticidade e gerando um ganho de massa após uma hora de imersão.
\end{abstract}

Palavras-chave: Colágeno. Adesivos Dentinários. Polifenóis. Proantocianidinas.

\begin{abstract}
Introduction: The use of different polyphenols in the increase of the mechanical properties of dentin collagen has been widely used, thus, tannic acid being a polyphenol with very pronounced biological proprieties. Objective: to evaluate the biomodifying potential of tannic acid (AT) in different concentrations in dentin collagen. Methods: the factors under investigation were modulus of elasticity, measured by a three-point bending test ( $\mathrm{n}=10$ ), and mass variation $(\mathrm{n}=10)$, measured by means of a precision scale, being the following substances evaluated: tannic acid $(0.1,1$ and $10 \%)$, proanthocyanidin $(6.5 \%)$ and distilled water (control). Results: As for the modulus of elasticity, the groups treated with tannic acid presented statistically higher values ( $p<0.05)$ in relation to the other groups, and no differences were observed between the different concentrations. For the values of mass variation, the groups that were immersed in biomodifiers showed statistically $(\mathrm{p}<0.05)$ values higher than the control group. Conclusion: tannic acid presents as a potential biomodifying agent of dentin collagen, regardless of the concentration used, increasing its modulus of elasticity and generating a mass gain after one hour of immersion.
\end{abstract}

Key words: Collagen. Dentin-Bonding Agents. Polyphenols. Proanthocyanidins

\section{INTRODUÇÃO}

Um dos maiores desafios em Odontologia restauradora é o estabelecimento de uma união eficaz entre sistemas resinosos e estrutura dentária. Em longo prazo, essa união pode não ser tão eficaz, em substrato dentinário, quando se trata de sistemas adesivos convencionais, pois, na interface de união adesivo/restauração, podem ocorrer diversos mecanismos de degradação por ação de enzimas metaloproteinases de matriz (MMPs) e cisteíno catepsinas (CTPs), além do processo de hidrólise, ocasionado por presença de água em excesso ${ }^{1,2,3}$. Já tem sido relatado, na literatura, que a taxa anual de falha de restaurações com resinas compostas varia em torno de 1 a $10 \%$, sendo a principal causa a presença de cáries secundárias oriundas de falhas no procedimento restaurador/adesivo ${ }^{4}$. Visando minimizar danos decorrentes dessas degradações, a literatura propõe a utilização de várias estratégias, como uso de inibidores de MMPs e CTPs, agentes remineralizadores, técnica úmida utilizando o etanol e os agentes biomodificadores ( $A B$ ) ou de ligações cruzadas $(A L C)^{5}$.

Os ALCs consistem em compostos químicos de origem natural ou sintética que irão interagir com o colágeno dentinário, formando ligações entre os aminoácidos presentes em sua estrutura tripla hélice, as quais podem ser tanto intrafibrilar como interfibrilar ${ }^{5}$. Por meio dessa ação, os ABs irão aumentar as propriedades mecânicas do colágeno da dentina, bem como inibir MMPs e $\mathrm{CTPs}^{5,6}$. Alguns ALCs não têm o seu mecanismo de ação bem descrito na literatura, como as proantocianidinas (PA), mas a hipótese mais bem aceita é que

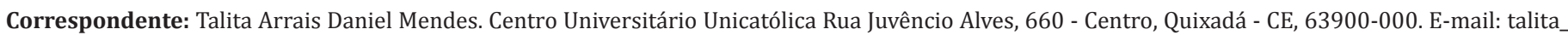
arrais@hotmail.com

Conflito de interesse: Não há conflito de interesse por parte de qualquer um dos autores.

Recebido 25 Out 2018; Revisado: 21 Jan 2019; 25 Jan 2019; Aceito: 28 Jan 2019 
o grupamento fenólico, presente em sua estrutura, irá interagir com os aminoácidos do colágeno ${ }^{5}$. Portanto, diversos outros compostos com característica semelhantes têm sido propostos como potenciais $A B$, sendo, em sua maioria, polifenóis. Tais compostos são descritos como moléculas fenólicas de origem natural, apresentando uma ou mais hidroxilas ligadas ao anel benzênico. São caracterizados por terem uma ampla atividade biológica, apresentando propriedades anti-inflamatórias, antioxidantes, anticancerígenas e influenciando diretamente na atividade de algumas enzimas e proteínas ${ }^{7}$.

Quando esses compostos interagem com o ácido gálico, por meio de uma reação de esterificação, irão formar ésteres que possuem atividade bem mais pronunciada ${ }^{8}$. Existem proposições de que a presença de grupamentos galoil em polifenóis melhora a capacidade de estabilização do colágeno, justificado pela maior quantidade de hidroxilas, as quais irão fornecer mais sítios reativos para que ocorra uma melhor reticulação do colágeno, podendo ocasionar sua maior rigidez, melhorando suas propriedades mecânicas ${ }^{9}$. Essa atividade biomodificadora melhorada irá depender, também, da posição e distribuição dos grupamentos galoil na molécula 10. Dessa forma, moléculas como o ácido tânico (AT), polifenol da família dos galotaninos, que apresentam cinco grupamentos galoil em sua estrutura, podem ser um potencial ALC de colágeno, tendo em vista as ótimas propriedades biológicas já conhecidas em sua molécula, excelente capacidade de inibição enzimática e reatividade biológica, eliminando radicais livres ${ }^{7}$.

A atividade dessas moléculas depende tanto da posição dos grupos galoil em sua estrutura, como de sua quantidade 9 . Dessa forma, o presente estudo objetiva avaliar a capacidade biomodificadora do ácido tânico, em diferentes concentrações no colágeno dentinário. As hipóteses do estudo foram (1) quão maior a concentração do ácido tânico, mais irá aumentar as propriedades mecânicas do colágeno tipo I e (2) conforme o aumento da concentração desse agente, maior será o ganho de massa após o tratamento do colágeno.

\section{MÉTODOS}

O presente estudo foi aprovado pelo Comitê de Ética em Pesquisa da Universidade Federal do Ceará (sob o número de protocolo 2439067). Trata-se de um estudo laboratorial in vitro, em que foram utilizados terceiros molares humanos livres de cárie para avaliar a dentina pré-tratada com AT 0,1 p/p\% (pó, Ácido Tânico, Sigma Aldrich, St. Louis, USA) [TA0,1]; AT 1p/p\% [TA1]; AT 10p/p\% [TA10]; extrato de uva contento incorporação de PA 6,5\% (90\% PA, Vitis vinifera, Gold Mega-Natural, polifenóis, Madera, USA) $[\mathrm{PA} 6,5]$ e água destilada [AD].

\section{Preparo das amostras}

Foram realizados cortes de dentina média de modo a obteremse barras com as dimensões de $1,7 \times 0,5 \times 6( \pm 0,1) \mathrm{mm}^{3}$. Esse corte foi realizado com o auxílio de uma cortadeira metalográfica sob constante refrigeração (Isomet 4000; Buehler, Lake
Bluff, Estados Unidos). As amostras foram desmineralizados completamente em solução de ácido fosfórico a $10 \%$ durante o período de 5 horas, em temperatura ambiente sob agitação. Todos os agentes de biomodificação foram diluídos em água deionizada na concentração pertinente a cada grupo com o auxílio de um agitador magnético até sua completa diluição, seguido de filtragem da solução. As barras de dentina foram distribuídas, aleatoriamente, em seus cinco grupos distintos $(n=10)$ e mantidas em suas respectivas soluções por um período de uma hora 10.

\section{Módulo de elasticidade (ME)}

O ME foi determinado em um ensaio de flexão de três pontos com uma célula de carga de 5,0 N montada em uma máquina de ensaios mecânicos universal (EMIC DL 2000, São José dos Pinhais, Brasil), utilizando-se a velocidade de $0,5 \mathrm{~mm} / \mathrm{min}$. Os dados foram expressos em Megapascal (MPa), sendo o aumento do ME calculado entre a razão do valor final (após a biomodificação da dentina nos respectivos grupos) e os valores iniciais ("baseline") ${ }^{10,11}$.

\section{Variação de massa (VM)}

Foram aferidas as massas dos espécimes antes (M1) e após (M2) a utilização dos agentes de biomodificação, com uma balança analítica de precisão de cinco casas decimais $(0,00001 \mathrm{~g})$. As amostras foram dessecadas a vácuo, durante 24 horas, à temperatura ambiente. A avaliação da variação de massa (VM\%) foi determinada como a porcentagem de ganho ou perda de base de cada amostra com base na seguinte fórmula:

$$
\mathrm{VM} \%=\left(\frac{\mathrm{M} 2 \times 100}{\mathrm{M} 1}\right)-100
$$

em que M1 é a massa matriz de dentina desmineralizada antes da biomodificação dentinária e M2 é a massa de matriz da dentina biomodificada 10.

\section{Análise estatística}

Realizou-se um teste de normalidade Shapiro-Wilk tanto para $\mathrm{ME}$, como para VM. Na avaliação do $\mathrm{ME}$, foi realizado o teste não paramétrico de Kruskal-Wallis, seguido de um pós-teste de Dunn's. Enquanto, para a VM, foi realizada uma análise de variância a um critério (ANOVA) seguido de pós-teste de Tukey. O nível de significância adotado para as duas análises foi de $5 \%$ $(p<0,05)$.

\section{RESULTADOS}

$\mathrm{Na}$ análise de $\mathrm{ME}$, pode-se observar que houve diferença estatística entre os grupos testados $(p<0,001)$, em que se pode observar que os grupos AT0,1; AT1 e AT10 apresentaram um ganho maior no ME quando comparados aos demais (tabela 1). Em relação à $V M$, pode-se observar que houve diferença estatística pelos grupos testados $(p<0,001)$. 0 grupo AD mostrou ganho de massa reduzido, quando comparados aos demais que apresentaram um ganho significativo de massa (tabela 1). 
Tabela 1. Dados expressos em médias das variações de massa (VM \%) e módulo de elasticidade (ME) [ \pm desvio-padrão] após desmineralização das barras de dentina seguidos prétratamento com as respectivas soluções por 1 hora.

\begin{tabular}{lrr}
\hline Grupos & ME & VM \\
\hline AT0,1 & $1,5 \pm 0,9 \mathrm{~A}$ & $6,9 \pm 0,5 \mathrm{~A}$ \\
AT1 & $7,9 \pm 6,5 \mathrm{~A}$ & $8,5 \pm 7,9 \mathrm{~A}$ \\
AT10 & $1,9 \pm 1,5 \mathrm{~A}$ & $10,5 \pm 1,9 \mathrm{~A}$ \\
PA6,5 & $-16,3 \pm 29,2 \mathrm{~B}$ & $8,5 \pm 2,3 \mathrm{~A}$ \\
AD & $-23,4 \pm 10,8 \mathrm{~B}$ & $3,1 \pm 1,3 \mathrm{~B}$ \\
\hline
\end{tabular}

*Para o ME, foi realizado um teste não paramétrico de Kruskal-Wallis, seguido de um pós-teste de Dunn's, e, para a VM, realizou-se uma análise de variância ANOVA a um critério seguido de um pós-teste de Tukey $(p<0,05)$. Nas letras maiúsculas - comparação entre colunas observa-se diferença estatística significativa, em que o ácido tânico 0,1\% (AT0,1), ácido tânico 1\% (AT1), ácido tânico 10\% (AT10\%), proantocianidina $6,5 \%(\mathrm{PA} 6,5)$ e água destilada como controle.

\section{DISCUSSÃO}

Não há um consenso na literatura a respeito do $A B$ ideal para aumentar a durabilidade da interface adesiva, evitando a perda de restaurações em um curto período de tempo ${ }^{5,6,11}$. Mas é uma realidade o aumento da utilização de produtos naturais na melhora da camada híbrida ${ }^{5,6,8,10}$, sendo o AT encontrado em algumas plantas da família Reaumuria e Tamarix ${ }^{12}$. O AT é um polifenol que apresenta propriedades biológicas bastante pronunciadas, em virtude da presença dos grupamentos galoil em sua molécula ${ }^{6,7,12}$, o que o caracteriza como um potencial agente biomodificador de colágeno dentinário. Dessa forma, o seu uso nesse substrato ainda não foi bem elucidado na literatura.

O uso do AT em diferentes concentrações aplicados sob a dentina modificou, positivamente, o colágeno, aceitando a primeira hipótese do estudo. O AT, independentemente da sua concentração, foi mais eficaz no aumento do ME que a PA, que é "padrão ouro" entre os AB naturais, ${ }^{5,13}$. As PAs já se mostram bastante eficazes na ligação com o colágeno tipo I presente na dentina, melhorando a conformação das fibrilas de colágeno, quando utilizada na concentração de $6,5 \%$. Tais substâncias apresentam uma versatilidade na formação de ligações, que podem ser do tipo ponte de hidrogênio, tipo éster ou, até mesmo, formação covalente estável entres os aminoácidos constituintes da cadeia tripla-hélice do colágeno ${ }^{5,13}$. Sabe-se, também, que, além de todos esses mecanismos de interação, tais catequinas irão formar complexos de cálcio amorfo, mineralizando tal estrutura por meio da deposição de fosfato e cálcio, deixando mais resistente o sítio de inserção do material restaurador ${ }^{14}$.

Já foi testada diferentes concentrações de AT (1\%, 10\% e 20\%) e observou-se que todas as concentrações influenciaram positivamente no módulo de elasticidade de colágeno dentinário quando aplicados por uma hora ${ }^{15}$. No entanto, não se obtiveram comparações com a proantocianidina, que, até então, já está bem estabelecida como um dos melhores agentes de reticulação de colágeno de origem natural, em estudos laboratoriais. $O$ presente estudo ainda demonstra que uma pequena concentração de AT, $0,1 \%$, foi eficaz no aumento do módulo de elasticidade do colágeno dentinário, mostrando o grande potencial de formação de ligações químicas com a estrutura dental. Dessa forma, nossos achados vão de encontro com Bedran-Russo et al (2009) que mostrou que as propriedades mecânicas do colágeno é dependente do tempo de aplicação da concentração ${ }^{15}$.

Aydin et al (2018) demonstraram que a presença de grupamentos galoil na molécula de proantocianidina galoilada não tem influência no aumento da longevidade do ganho de propriedades mecânicas do colágeno tratado, sendo a PAC sem esses grupamentos mais eficaz nessa manutenção 16 , dessa forma, o presente estudo analisou de maneira imediata esse colágeno pré-tratado, e os grupamentos galoil parecem ter sido relevantes na formação de ligações cruzadas no colágeno de dentina; no entanto, faz-se necessária uma avaliação em longo prazo para saber se essas ligações químicas possuem estabilidade com o passar o tempo .

Sendo assim, observou-se que o AT independente da concentração utilizada, foi mais eficaz que a PA, e isso pode verse pelo fato de a molécula do TA ter maior sítios de provável reticulação com o colágeno, apresentando uma ampla atividade biológica, bem como formar maior número de ligações cruzadas que irão inibir, de maneira mais efetiva, as MMPs e CTPs, preservando, efetivamente, a estrutura colágena ${ }^{6,7,11,15}$. Por ser uma complexa mistura de poligaloilglucose, terá, consequentemente, uma maior quantidade de hidroxilas disponíveis para interação com o grupamento amida, que são constituintes dos aminoácidos presentes na tripla hélice do colágeno dentinário, corroborando os achados que sugerem que a quantidade de hidroxilas está diretamente relacionada à reatividade e à interação dessa molécula na efetividade de ligações cruzadas ${ }^{8,15}$.

Quando analisamos os três grupos contendo AT, podemos observar que, na concentração de $10 \%$, há um menor ganho desse $\mathrm{ME}$, comparado aos demais. Isso pode dar-se pelo fato da não completa interação do substrato em questão, que apresenta, consequentemente, moléculas residuais que podem interferir em uma posterior ligação com sistemas adesivos. Isso pode ser observado nas PAs, que, por não apresentarem uma pureza total no extrato de uva, os resquícios existentes vão influenciar negativamente para o ganho de suas propriedades mecânicas.

Além do aumento do ME demonstrado no presente estudo, observa-se uma característica favorável para sua implementação na prática clínica, que é a influência na coloração, que as PAs pigmentam fortemente o substrato ${ }^{17}$, o que não é observado nos grupos usando TA, o que viabiliza o seu uso na odontologia estética, tornando-o bastante promissora a sua aplicabilidade. 
Em relação à variação de massa, observou-se diferença significativamente estatística entre os grupos testados, aceitando a segunda hipótese do estudo. Já é bem estabelecida, na literatura, a bioatividade de algumas moléculas como taninos e flavonoides ${ }^{6,10,11}$, e isso pode ser comprovado pelo ganho de massa nos grupos AT0,1, AT1, AT10 e PA6,5, referente à formação de ligações cruzadas na estrutura colágena, comprovando a efetividade de AT com essa função para melhorar a interface adesiva.

Alguns achados, na literatura, já mostram que, na concentração $6,5 \%$, a PA é mais eficaz na efetividade de melhora das propriedades mecânicas da dentina pré-tratada ${ }^{5,14,15}$, corroborando o presente estudo que mostrou um ganho significativo de massa equivalente à PA, elucidando a atividade de ALC desses. No entanto, o mecanismo de ação, o AT, ainda não está bem estabelecido, necessitando de maiores estudos acerca desse questionamento. Portando, acredita-se que o AT vai melhorar o ME e o ganho de massa por meio de formação de ligações cruzadas.

\section{CONCLUSÃO}

Conclui-se que o ácido tânico influencia, positivamente, no ganho de propriedades mecânicas do colágeno tipo I, presente na dentina, e aumenta, significativamente, a massa da dentina desmineralizada, independentemente da concentração utilizada, na aplicação de 1hora, sendo então, eficaz para a formação de ligações cruzadas, podendo ser promissor na utilização perante o procedimento adesivo.

\section{AGRADECIMENTOS}

À Coordenação de Aperfeiçoamento de Pessoal de Nível Superior e à Universidade Federal do Ceará.

\section{REFERÊNCIAS}

1. Pashley DH, Tay FR, Breschi L, Tjaderhane L, Carvalho RM, Carrilho M, Tezvergil-Mutluay A. State of the art etch-and-rinse adhesives. Dent Mater [Internet]. 2011 Jan [cited 2018 Out 21]; 27(1):1-16. Avaliable from: https:// www.ncbi.nlm.nih.gov/pmc/articles/pmid/21112620/. doi: 10.1016/j. dental.2010.10.016

2. Frasseto A, Breschi L, Turco G, Marchesi G, Di Lenarda R, Tay FR, Pashley $\mathrm{DH}$, Cadenaro $\mathrm{M}$. Mechanisms of degradation of the hybrid layer in adhesive dentistry and therapeutic agents to improve bond durability:A literature review. Dent Mater [Internet]. 2016 Fev [cited 2018 Out 22]. 32(2): 41-53. Avaliable from: https://linkinghub.elsevier.com/retrieve/pii/S0109-5641(15)00465-0. doi: 10.1016/j.dental.2015.11.007.

3.Niu L, Zhang W, Pashley DH, Breschi L, Mao J, Chen J, Tay FR. Biomimetic remineralization of dentin. Dent Mater [Internet]. . 2014 Jan [cited 2018 Out 22]30(1): 77-96. Avaliable from: https://linkinghub.elsevier.com/retrieve/pii/ S0109-5641(13)00175-9. doi: 10.1016/j.dental.2013.07.013.

4. Demarco FF, Corrêa MB, Cenci MS, Moraes RR, Opdam NJM. Longevity of posterior composite restorations: Not only a matter of materials. Dent Mater [Internet]. 2012 Jan [cited 2019 Jan 21]. 28(1): 87-101. Avaliable from: www. intl.elsevierhealth.com/journals/dema. doi:10.1016/j.dental.2011.09.003.

5. Bedran-Russo AK, Pauli GF, Chen SN, Mcalpine J, Castellan CS, Phansalkar RS, et al. Dentin biomodification: strategies, renewable resources and clinical applications. Dent Mater [Internet] . 2014 Out [cited 2018 Out 22]. 30(1): 62-76. Avaliable from: https://www.ncbi.nlm.nih.gov/pmc/articles/pmid/24309436/. doi: 10.1016/j.dental.2013.10.012.

6. Vidal CMP, Aguiar TR, Phansalkar R, Mcalpine JB, Napolitano JG, Chen SN, et al. Galloyl moieties enhance the dentin biomodification potential of plantderived catechins. Acta Biomater [Internet]. 2014 Abr [cited 2018 Out 22].10(7): 3288-3294. Avaliable from: https://www.ncbi.nlm.nih.gov/pmc/articles/ pmid/24721612/. DOI: 10.1016/j.actbio.2014.03.036.

7. Karas D, Ulrichová J, Valentová K. Galloylation of polyphenols alters their biological activity. Food Chem Toxicol [Internet]. 2017 Jul [cited 2018 Out 22]. 105(12): 223-240. Avaliable from: https://linkinghub.elsevier.com/retrieve/pii/ S0278-6915(17)30188-6. doi: 10.1016/j.fct.2017.04.021.

8. Phansalkar RS, Nam JW, Chen SN, Mcalpine JB, Napolitano JG, Leme A, Vidal $\mathrm{CMP}$, et al. A galloylated dimeric proanthocyanidin from grape seed exhibits dentin biomodification potential. Fitoterapia [Internet]. 2015 Dez [cited 2018 Out 22]. 101(2):168-178. Avaliable from: https://www.ncbi.nlm.nih.gov/pmc/ articles/pmid/25542682/. doi: 10.1016/j.fitote.2014.12.006.

9. He Q, Shi B, Yao K, Luo Y, Ma Z. Synthesis of gallotannins. Carbohydr Res [Internet]. 2001 Oct [cited 2018 Out 22]. 335(4): 245-250. Avaliable from: https://linkinghub.elsevier.com/retrieve/pii/S0008621501002361. doi: 10.1016/S0008-6215(01)00236-1.

10. Aguiar TR, Vidal CMP, Phansalkar RS, Todorova I, Napoliatano JG, Mcalpine JB, Chen SN, Pauli GF, Bedran-Russo AK. Dentin Biomodification Potential Depends on Polyphenol Source. J Dent Res [Internet]. 2014 Set [cited 2018 Out 22]. 93(4): 417-422. Avaliable from: https://www.ncbi.nlm.nih.gov/pmc/ articles/pmid/24574140/. doi: 10.1177/0022034514523783.

11. Castellan CS, Pereira PNR, Viana G, Chen SN, Pauli GF, Bedran-Russo AK. Solubility study of phytochemical cross-linking agents on dentin stiffness. J Dent [Internet]. 2010 Feb [cited 2018 Out 22]. 38(5): 431-436. Avaliable from: https://www.ncbi.nlm.nih.gov/pmc/articles/pmid/20171257/. doi: 10.1016/j. jdent.2010.02.002.

12. Orabi MA, Yoshimura M, Amakura Y, Hatano T. Ellagitannins, gallotannins, and gallo-ellagitannins from the galls of Tamarix aphylla. Fitoterapia [Internet]. 2015 Jul [cited 2018 Out 22]. 104:55-63. Avaliable from: https://linkinghub.elsevier. com/retrieve/pii/S0367-326X(15)30004-6. doi: 1 10.1016/j.fitote.2015.05.008.

13. Vidal CMP, Zhu W, Manhoar S, Aydin B, Keiderling TA, Messersmith PB, Bedran-Russo AK. Collagen-collagen interactions mediated by plant-derived proanthocyanidins: A spectroscopic and atomic force microscopy study. Acta Biomater [Internet]. 2016 May [cted 2018 Out 21]. 41:110-118. Avaliable from: https://www.ncbi.nlm.nih.gov/pmc/articles/pmid/27208639/. doi: 10.1016/j. actbio.2016.05.026

14. Epasinge DJ, Burrow MF, Yiu CKY. Effect of proanthocyanidin on ultrastructure and mineralization of dentin collagen. Arch Oral Biol [Internet]. 2017 Dec [cited 2018 Out 22]. 84: 29-36. Avaliable from: https://linkinghub.elsevier.com/ retrieve/pii/S0003-9969(17)30283-2. doi: 10.1016/j.archoralbio.2017.09.012.

15. Bedran-Russo AKB, Yoo KJ, Ema KC, Pashley DH. Mechanical Properties of Tannic-acid-treated Dentin Matrix. J Dent Res [Internet]. 2009 Sep [cited 2018 Oct 21]. 88(9): 807-811. Avaliable from: https://www.ncbi.nlm.nih.gov/pmc/ articles/pmid/19767576/. doi: 10.1177/0022034509342556.

16. Aydin B, Leme-Kraus AA, Vidal CMP, Aguiar TR, Phasalkar RS, Nam JW, et al. Evidence to the role of interflavan linkages and galloylation of proanthocyanidins at sustaining long-term dentin biomodification. Dent Mater [Internet]. $2018 \mathrm{Dec}$ 
[cited 2019 Jan 21]. 35(2): 328-334. Avaliable from: www.intl.elsevierhealth. com/journals/dema. doi: 10.1016/j.dental.2018.11.029.

17. Moreira MA, Souza NO, Sousa RS, Freitas DQ, Lemos MS, De Paula DM, et al. 2017 Oct [cited 2018 Oct 21]. Efficacy of new natural biomodification agentsfrom Anacardiaceae extracts on dentin collagencross-linking. Dent Mater [Internet]. 33(10):1103-1109. Avaliable from: https://linkinghub.elsevier.com/ retrieve/pii/S0109-5641(17)30320-2/. doi: 10.1016/j.dental.2017.07.003.

\section{Como citar este artigo/How to cite this article:}

Mendes TAD, Pascoal SCD, Lemos MVS, Santiago SL, Mendonça JS. Ácido tânico como agente biomodificador de dentina. J Health Biol Sci. 2019 Abr-Jun; $7(2): 182-186$ 